\title{
Modeling of Damped Vibrations of Cantilevered Piezoelectric Actuators
}

\author{
Dan Liao ${ }^{1}$, Yanhui Yuan ${ }^{1,2}$, Kunchao Yang $^{3}$, Hao Liang $^{3}$, Xiaoqin Gu ${ }^{1}$ \\ ${ }^{1}$ University of Electronic Science and Technology of China, Zhongshan Institute, Zhongshan, Guangdong, 528400 China \\ ${ }^{2}$ Zhongshan Branch of State Key Laboratory of Electronic Thin Films and Integrated Devices, Zhongshan, Guangdong, 528400 China \\ ${ }^{3}$ University of Electronic Science and Technology of China, School of Mechanical \& Electrical Engineering, Chengdu, Sichuan, 611731 \\ China
}

\begin{abstract}
Damped vibrations of cantilevered piezoelectric actuators were modeled and analyzed. The differential equations of motion derived by the extended Hamilton's principle were a boundary-value problem with nonhomogeneous boundary conditions. An equivalent boundary-value problem with homogeneous boundary conditions was established through transformation and solved by modal analysis. Responses in time and frequency domains for unimorph and bimorph were obtained. Frequency response functions under excitations of voltage and tip force were verified by three-dimensional finite element method (FEM). Discrepancies between the analytical and FEM results were within 3\%. Effects of distributed viscous damping were investigated using the analytical model. Numerical results showed that the vibration amplitude and resonant frequencies changed in opposite directions with increasing damping. This phenomenon was also reported in literature on generic cantilevers of a single material.
\end{abstract}

\section{Introduction}

In previous studies, differential equations of motion for piezoelectric cantilevers were usually derived based on generic cantilevers of a homogeneous material. The piezoelectric moment induced by voltage was incorporated either in the differential equations [1] or in the boundary conditions [2].

Although piezoelectric cantilevers are similar to generic cantilevers in terms of dynamic behavior under external loading, some assumptions of generic cantilevers may not be applicable to multilayer piezoelectric cantilevers under excitation of voltage. Therefore, it is desirable to start with a more fundamental approach without relying on models of generic cantilevers as a basis. For this purpose, Tanaka [3] and Ballas [4] used Hamilton's principle, a powerful variational principle of mechanics, to systematically derive the equations of motion, charge equation of electrostatics and boundary conditions for multilayer piezoelectric cantilevers. Both Tanaka and Ballas's derivations yielded the piezoelectric moment in the boundary conditions. However, the nonhomogeneous boundary conditions caused difficulty for modal analysis [5]. Tanaka [3] did not solve the differential equations in his study. Ballas [4] attempted solution by modal analysis; however, the piezoelectric moment was moved from the boundary conditions to the right hand of the differential equations by treating it as a load per unit length.

The aim of the present study was to model damped vibrations of cantilevered piezoelectric actuators in a systematic and rigorous manner. The extended Hamilton's principle was used to derive the differential equations of motion. Proportional damping was considered to enable diagonalization of the damping matrix. To solve the partial differential equations with nonhomogeneous boundary conditions by modal analysis, the boundary-value problem was transformed into a new problem with homogeneous boundary conditions. Modal analysis of the new problem would then lead to analytical solutions to damped vibration of multilayer piezoelectric cantilevers. The analytical solutions were compared with finite-element-method (FEM) results. Finally, a parametric study using the analytical model was conducted to investigate effects of distributed viscous damping on vibration amplitude, phase angle and resonant frequencies.

\section{Mathematical formulation of cantilevered piezoelectric actuators}

Figure 1 shows the model of cantilevered multilayer piezoelectric actuators subjected to time varying loadings such as uniform pressure $f(x, t)$, concentrated force $F_{c}(t)$ and tip moment $M(t)$. The cantilever can operate as either an actuator or a sensor depending on whether voltage $V(t)$ is applied as a source or measured as a signal. Unimorphs and bimorphs are two most common versions of multilayer piezoelectric cantilevers. A unimorph consists of a single piezoelectric layer on top of an elastic substrate, while a bimorph two 
piezoelectric layers with or without a sandwiched elastic substrate.

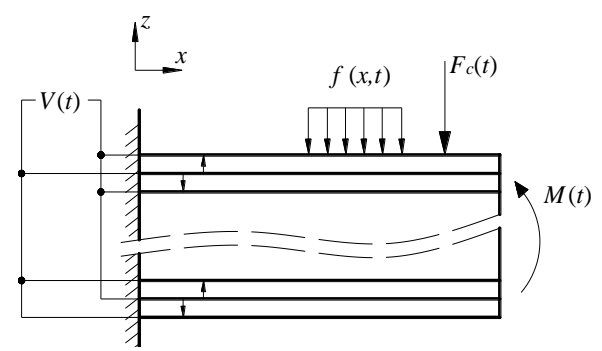

Figure 1. Model of cantilevered multilayer piezoelectric actuators.

\subsection{Derivation of differential equations of motion}

For a slender cantilever beam, stress components other than the axial stress are negligible. Thus, the standard three-dimensional piezoelectric constitutive equations [6] can be reduced to a one-dimensional form

$$
\left[\begin{array}{l}
S_{1} \\
D_{3}
\end{array}\right]=\left[\begin{array}{ll}
s_{11}^{E} & d_{31} \\
d_{31} & \varepsilon_{33}^{T}
\end{array}\right]\left[\begin{array}{l}
T_{1} \\
E_{3}
\end{array}\right]
$$

where $S_{1}$ is the axial strain in the x-direction, $D_{3}$ electric displacement in the z-direction, $s_{11}^{E}$ elastic compliance at constant electric field, $d_{31}$ piezoelectric coupling coefficient, $\varepsilon_{33}^{T}$ permittivity at constant strain, $T_{1}$ axial stress in the x-direction, $E_{3}$ electric field in the zdirection, respectively. Considering the kinetic energy, potential energy, and work done by external and nonconservative forces, the extended Hamilton's principle yields [3-5]

$$
\begin{aligned}
& \int_{t_{1}}^{t_{2}} d t\left\{\int_{0}^{t}\left[-m \frac{\partial^{2} w}{\partial t^{2}}-K \frac{\partial^{4} w}{\partial x^{4}}-\left(C_{s} K \frac{\partial w^{5}}{\partial x^{4} \partial t}+C_{a} \frac{\partial w}{\partial t}\right)+f(x, t)+F_{c}(t) \delta\left(x-x_{c}\right)\right]\right. \\
& \delta w d x+\sum_{n=1}^{N} b_{n} \int_{z_{n}}^{z_{n+1}} \int_{0}^{t} \frac{d D_{3, n}}{d z} \delta V d x d z+\left.K \frac{\partial^{3} w}{\partial x^{3}} \delta w\right|_{0} ^{l}-\left[K \frac{\partial^{2} w}{\partial x^{2}}-M_{P}(t)-M(t)\right] \\
& \left.\left.\delta \frac{\partial w}{\partial x}\right|_{0} ^{l}-\sum_{n=1}^{N} b_{n}\left[\int_{0}^{l}\left(\sigma_{n}+D_{3, n}\right) d x \delta V \mid \begin{array}{c}
z_{n+1} \\
z_{n}
\end{array}\right]\right\}=0
\end{aligned}
$$

where $t_{1}, t_{2}$ are initial and final times, $m$ mass density per length, $K$ bending stiffness, $C_{s}$ internal material damping coefficient, $C_{a}$ viscous damping coefficient, $\delta\left(x-x_{c}\right)$ a Dirac delta function, $b_{n}$ width of the $n$-th layer, $M_{p}(\mathrm{t})$ piezoelectric moment and $\sigma_{n}$ surface charge per unit area for the $n$-th layer, respectively. The bending stiffness $K$ is given by

$$
K=\sum_{n=1}^{N} \frac{1}{s_{11, n}} \int_{z_{n}}^{z_{n+1}} b_{n}\left(z-z_{0}\right)^{2} d z
$$

where $z_{0}$ is the $\mathrm{z}$-coordinate of the neutral plane. The piezoelectric moment $M_{p}(t)$ is written as

$$
M_{P}(t)=\sum_{n=1}^{N} \frac{b_{n} V_{n} d_{31, n}}{2 s_{11, n}^{E}}\left(z_{n+1}+z_{n}-2 z_{0}\right)
$$

where $V_{n}$ is the voltage applied to the $n$-th layer.

In Eq. (2), the virtual displacement $\delta w$ is arbitrary. Hence,

$$
m \frac{\partial^{2} w}{\partial t^{2}}+C_{s} E I \frac{\partial w^{5}}{\partial x^{4} \partial t}+C_{a} \frac{\partial w}{\partial t}+K \frac{\partial^{4} w}{\partial x^{4}}=f(x, t)+F_{c}(t) \delta\left(x-x_{c}\right)
$$

Similarly, we obtain the boundary conditions for the piezoelectric cantilever as

$$
w(0, t)=0, \frac{\partial w(0, t)}{\partial x}=0
$$

and

$$
K \frac{\partial^{3} w(l, t)}{\partial x^{3}}=0, K \frac{\partial^{2} w(l, t)}{\partial x^{2}}=M_{P}(t)+M(t)
$$

where $l$ is the length of the piezoelectric cantilever.

\subsection{Transformation of the boundary-value problem}

The solution to boundary-value problems consisting of nonhomogeneous differential equations of motion and homogeneous boundary conditions is usually obtained by modal analysis. For boundary-value problems with nonhomogeneous boundary conditions, the common approach used in modal analysis does not work. However, a boundary-value problem with nonhomogeneous boundary conditions can be transformed into a problem with homogeneous boundary conditions which can be solved by modal analysis [5]. We assume

$$
w(x, t)=v(x, t)+h(x)\left[M_{P}(t)+M(t)\right]
$$

where $v(x, t)$ is the solution to the transformed problem, $h(x)$ a function to be found to satisfy the homogeneous boundary conditions of the new problem, respectively.

Upon inserting Eq. (8), Eq. (5) becomes,

$K \frac{\partial^{4} v(x, t)}{\partial x^{4}}+C_{s} K \frac{\partial v^{5}}{\partial x^{4} \partial t}+C_{a} \frac{\partial v(x, t)}{\partial t}+m \frac{\partial^{2} v(x, t)}{\partial t^{2}}=Q(x, t)(9)$

where $Q(x, t)$ is expressed as

$$
\begin{aligned}
& Q(x, t)=f(x, t)+F_{c}(t) \delta\left(x-x_{c}\right)-m h(x)\left[\ddot{M}_{P}(t)+\ddot{M}(t)\right] \\
& -K \frac{d^{4} h(x)}{d x^{4}}\left[M_{P}(t)+M(t)\right]-C_{s} K \frac{d^{4} h(x)}{d x^{4}}\left[\dot{M}_{P}(t)+\dot{M}(t)\right] \\
& -C_{a} h(x)\left[\dot{M}_{P}(t)+\dot{M}(t)\right]
\end{aligned}
$$

To render all the boundary conditions of the new problem homogeneous, we must have

$$
v(0, t)=0, \frac{\partial v(0, t)}{\partial x}=0, \frac{\partial^{3} v(l, t)}{\partial x^{3}}=0, K \frac{\partial^{2} v(l, t)}{\partial x^{2}}=0
$$

$h(x)$ can be any arbitrary function satisfying the above boundary conditions. Assuming $h(x)$ is a 
polynomial and considering the $\frac{d^{4} h(x)}{d x^{4}}$ term in Eq. (10), $h(x)$ must be of an order equal to or higher than fifth. Hence, we find $h(x)$

$$
h(x)=-\frac{1}{10 K l^{3}} x^{5}+\frac{1}{4 K l^{2}} x^{4}
$$

The original boundary-value problem with nonhomogeneous boundary conditions has now been transformed into a new problem with homogeneous boundary conditions which can be solved by modal analysis.

\subsection{Frequency response functions}

Following the normal procedure of modal analysis, the modal shapes $Y_{r}(x)$, damping ratios $\zeta_{r}$ and modal coordinates can be obtained [5]. The frequency response functions for excitations of voltage $G_{V}(i \omega)$, tip moment $G_{M}(i \omega)$ and tip force $G_{F}(i \omega)$ are found and given by

$$
\begin{gathered}
G_{V}(i \omega)=\sum_{n=1}^{N} \frac{b_{n} d_{31, n}}{2 s_{11, n}^{E}}\left(z_{n+1}+z_{n}-2 z_{0}\right)[h(x)+ \\
\left.\sum_{r=1}^{\infty} \frac{Y_{r}(x) \int_{0}^{l} Y_{r}(x)\left(m h(x) \omega^{2}-C_{s} K \frac{d^{4} h(x)}{d x^{4}} i \omega-C_{a} h(x) i \omega-K \frac{d^{4} h(x)}{d x^{4}}\right) d x}{\omega_{r}^{2}-\omega^{2}+2 i \zeta_{r} \omega \omega_{r}}\right] \\
\sum_{r=1}^{\infty} \frac{Y_{M}(x) \int_{0}^{l} Y_{r}(x)\left(m h(x) \omega^{2}-C_{s} K \frac{d^{4} h(x)}{d x^{4}} i \omega-C_{a} h(x) i \omega-K \frac{d^{4} h(x)}{d x^{4}}\right) d x}{\omega_{r}^{2}-\omega^{2}+2 i \zeta_{r} \omega \omega_{r}} \\
G_{F}(i \omega)=\sum_{r=1}^{\infty} \frac{Y_{r}(x) Y_{r}(l)}{\omega_{r}^{2}-\omega^{2}+2 i \zeta_{r} \omega \omega_{r}}
\end{gathered}
$$

Comparison of Eq. (13) and Eq. (14) shows that the effect of the piezoelectric moment is equivalent to that of the external tip moment.

\section{Verification of the mathematical model}

The analytical solutions were verified by a commercial FEM program (Ansys Multiphysics 17.0). The dimensions of the two cantilevers are listed in Table 1.l, $b, t_{p}, t_{e}$ and $t_{s}$ are the length, width of the cantilever, thicknesses of the piezoelectric layers, electrodes and elastic substrate, respectively.

Table 1. Dimensions of cantilevered piezoelectric actuators.

\begin{tabular}{|c|c|c|c|c|c|}
\hline Dimensions & $\boldsymbol{l}(\mu \mathrm{m})$ & $\boldsymbol{b}(\mu \mathrm{m})$ & $\boldsymbol{t}_{\boldsymbol{p}}(\mu \mathrm{m})$ & $\boldsymbol{t}_{\boldsymbol{e}}(\mu \mathrm{m})$ & $\boldsymbol{t}_{\mathrm{s}}(\mu \mathrm{m})$ \\
\hline Unimorph & 2000 & 200 & 1 & 0.1 & 20 \\
\hline Bimorph & 2000 & 200 & 10 & 0.1 & 0 \\
\hline
\end{tabular}

The materials used for the electrodes, elastic substrate and piezoelectric layers were gold, silicon and zinc oxide ( $\mathrm{ZnO})$, respectively. Element type SOLID226 was used for the piezoelectric layers, SOLID186 for elastic substrate, and SHELL281 for electrodes. The length for both cantilevers was divided into 200 segments in the analytical and FEM models. Frequency responses to voltage and tip force were obtained by the analytical model and compared to the FEM results.

No damping was considered in the numerical results due to incompatible damping formulations in the present work and Ansys. In the mathematical model described by Eq. (9), material damping and viscous damping were represented by two constants, $C_{s}$ and $C_{a}$, respectively, whereas for harmonic analysis in Ansys, damping is expressed as a structural damping matrix which is assembled by stiffness and mass matrices and associated multipliers [7]. Equivalency between these two methods is yet to be established. In the plot of frequency response function, the magnitude in the lower frequency (quasistatic) range and the resonant frequency are largely independent of damping. Therefore, the accuracy of the analytical model could be evaluated by the undamped frequency response based on these two quantities.

\subsection{Frequency response function to voltage excitation}

Frequency response function of tip deflection of the unimorph excited by voltage is shown in Fig. 2. The first resonant frequencies were $6.58 \mathrm{kHz}$ and $6.64 \mathrm{kHz}$ for the analytical model and FEM model, respectively. The percent error was $0.9 \%$. For quasi-static responses, tip deflections at $200 \mathrm{~Hz}$ as seen in the inset were calculated. The magnitudes were $80.91 \mathrm{~nm}$ and $80.67 \mathrm{~nm}$ for the analytical model and FEM model, respectively. The percent error was $0.3 \%$. For the unimorph under voltage excitation, the undamped frequency response obtained by the analytical model was very close to the FEM results.

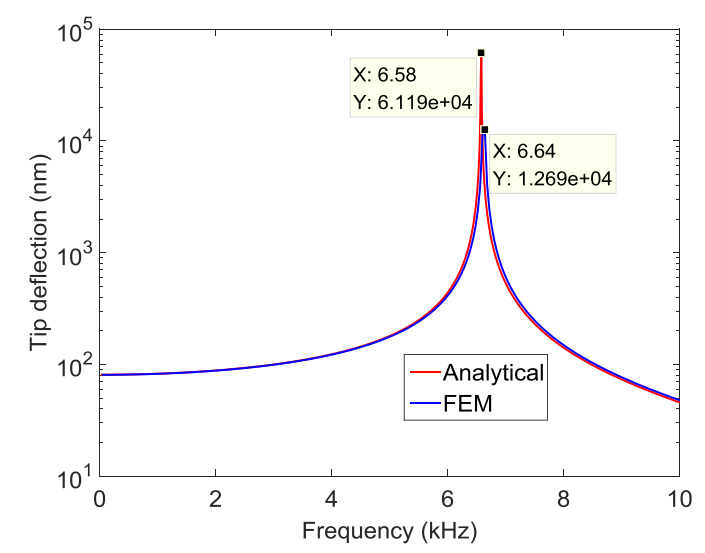

Figure 2. Frequency response function of unimorph to voltage excitation.

Frequency response function of tip deflection of the bimorph excited by voltage is shown in Fig. 3. The percent errors for the resonant frequency and tip deflection at $200 \mathrm{~Hz}$ were $1.2 \%$ and $0.7 \%$, respectively. The errors were slightly bigger than those of the unimorph. However, the accuracy of the analytical model remained very high. 


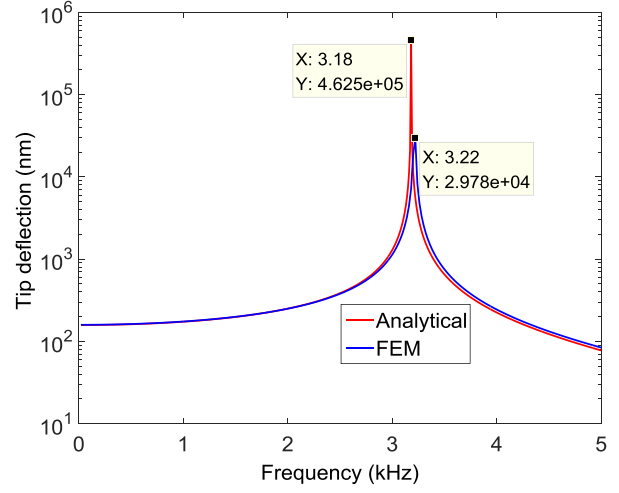

Figure 3. Frequency response function of bimorph to voltage excitation.

\subsection{Frequency response function to excitation of tip force}

The validity of the mathematical model was also verified for frequency response function to tip force. Frequency response functions of the unimorph and bimorph are plotted in Fig. 4 and Fig. 5, respectively. As seen in Fig. 4 , the resonant frequencies of the unimorph were exactly the same as those under voltage excitation (Fig. 2). For the bimorph, the mathematical model also produced the same results of $3.18 \mathrm{kHz}$ (Fig. 3 \& Fig. 5), while the FEM model had a small deviation, from $3.22 \mathrm{~Hz}$ to 3.24 $\mathrm{Hz}$, which could be eliminated by increasing the number of nodes.

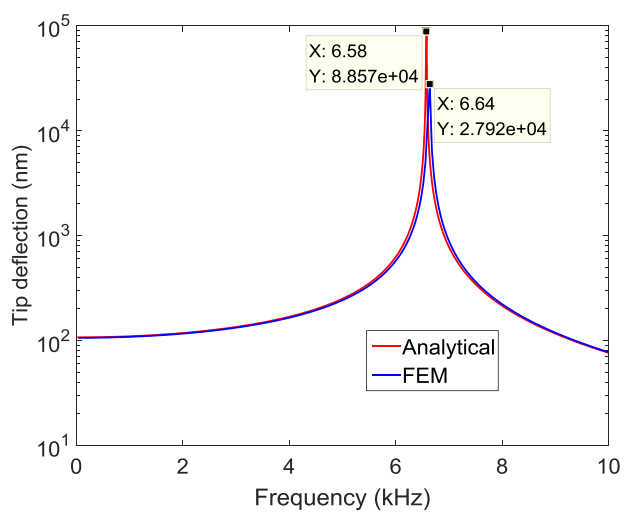

Figure 4. Frequency response function of unimorph to excitation of tip force.

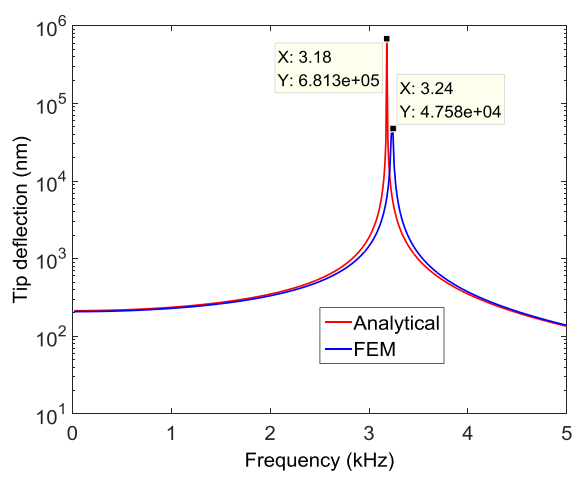

Figure 5. Frequency response function of bimorph to excitation of tip force.
The magnitudes at quasi-static state $(200 \mathrm{~Hz})$ of the unimorph were $107.3 \mathrm{~nm}$ and $106.1 \mathrm{~nm}$ for the mathematical model and FEM model, respectively. The corresponding percent error was $1.1 \%$. For the bimorph, the magnitudes were $214.1 \mathrm{~nm}$ and $208.3 \mathrm{~nm}$ for the mathematical model and FEM model, respectively. The percent error increased slightly to $2.8 \%$.

All results are listed in Table 2. The maximum percent error was $2.8 \%$ for the magnitude of the bimorph under excitation of tip force. The mathematical model was verified to be highly accurate.

Table 2. Summary of verification results.

\begin{tabular}{|c|l|c|c|c|c|}
\hline \multicolumn{2}{|c|}{} & \multicolumn{2}{c|}{$\begin{array}{c}\text { Voltage } \\
\text { excitation }\end{array}$} & \multicolumn{2}{c|}{$\begin{array}{c}\text { Tip force } \\
\text { excitation }\end{array}$} \\
\cline { 3 - 6 } \multicolumn{2}{|c|}{} & $\begin{array}{c}\boldsymbol{f}_{\mathbf{1}} \\
(\mathrm{kHz})\end{array}$ & $\begin{array}{c}\boldsymbol{\delta}_{\mathbf{2} 0 \mathrm{~Hz}} \\
(\mathrm{~nm})\end{array}$ & $\begin{array}{c}\boldsymbol{f}_{1} \\
(\mathrm{kHz})\end{array}$ & $\begin{array}{c}\boldsymbol{\delta}_{\mathbf{2 0 H}} \\
(\mathrm{nm})\end{array}$ \\
\hline \multirow{3}{*}{ Unimorph } & Analytical & 6.58 & 80.91 & 6.58 & 107.3 \\
\cline { 2 - 6 } & FEM & 6.64 & 80.67 & 6.64 & 106.1 \\
\cline { 2 - 6 } & Error (\%) & 0.9 & -0.3 & 0.9 & 1.1 \\
\hline \multirow{3}{*}{ Bimorph } & Analytical & 3.18 & 158.5 & 3.18 & 214.1 \\
\cline { 2 - 6 } & FEM & 3.22 & 159.6 & 3.24 & 208.3 \\
\cline { 2 - 6 } & Error (\%) & 1.2 & 0.7 & 1.9 & 2.8 \\
\hline
\end{tabular}

\section{Effects of viscous damping}

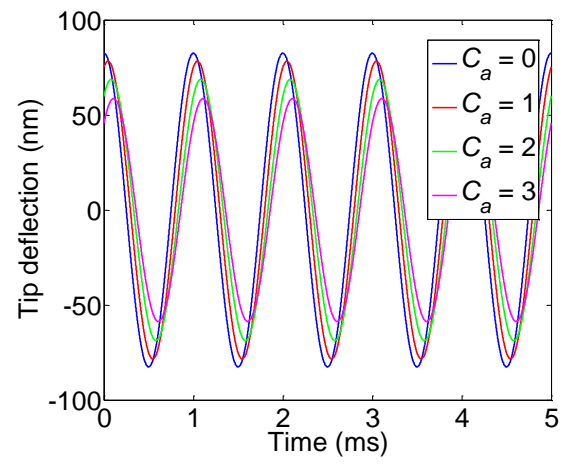

Figure 6. Steady-state time response of unimorph to sinusoidal voltage excitation of $1 \mathrm{~V}$ at $1000 \mathrm{~Hz}$.

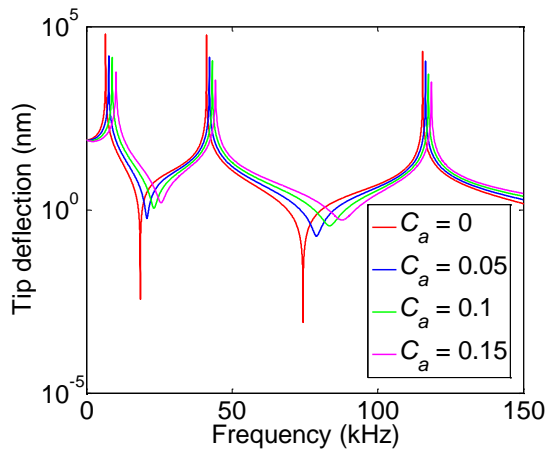

Figure 7. Effect of damping on frequency response function of unimorph to voltage excitation.

The effects of viscous damping on performance of the unimorph was studied with the analytical model. To consider only distributed viscous damping $\left(C_{a}\right), C_{s}$ in Eq. (5) was set to zero. Figure 6 shows the steady-state time response of the unimorph with different levels of damping. The amplitude of tip deflection decreased with 
damping, while the phase angle increased with damping. This phenomenon was in agreement with the behavior of single-degree-of-freedom (SDOF) systems.

However, as seen in Fig. 7 the resonant frequencies increased with damping which was contrary to SDOFsystems. This phenomenon also exists on generic cantilevers [8]. Considering similarities shared by generic and piezoelectric cantilevers, the effects of viscous damping further proved the validity of the mathematical model.

\section{Conclusions}

The constitutive equations for flexural vibration of multilayer piezoelectric cantilevers were derived by the extended Hamilton's principle. The piezoelectric moment was found in the boundary conditions alongside the external tip moment. The frequency response functions showed that the effect of the piezoelectric moment is equivalent to that of the external tip moment. The analytical solutions were verified with 3D FEM analysis. Deflection profiles and frequency response functions under voltage and tip force were compared. Discrepancies between the analytical and FEM results were within $2.8 \%$. Effects of distributed viscous damping on resonant frequencies were found to be contrary to vibration amplitude. This finding was in line with the same phenomenon of generic cantilevers

\section{Acknowledgement}

This work was funded by Natural Science Foundation of Guangdong Province (Grant No. 2015A030313680) and National Natural Science Foundation of China (Grant No. 61771118).

\section{References}

1. A. Erturk, D. J. Inman, J. Vib. Acoust. 130, 4 (2008)

2. M. Brissaud, S. Ledren, P. Gonnard, J Micromech. Microeng. 13, 6 (2003)

3. H. Tanaka, J. Acoust. Soc. Am. 95, 4 (1994).

4. R. G. Ballas, Piezoelectric multilayer beam bending actuators: static and dynamic behavior and aspects of sensor integration (Springer, New York, 2007)

5. L. Meirovitch, Principles and techniques of vibrations. (Prentice Hall, Upper Saddle River, N.J, 1997)

6. IEEE Standard on Piezoelectricity. (Institute of Electrical and Electronic Engineers, New York, 1988)

7. Theory Reference of Ansys 17.0 Documentation. (ANSYS Inc, 2016)

8. A. W. Leissa, M. S. Qatu, Vibrations of continuous systems. (McGraw-Hill, New York, 2011) 